\title{
Nanomedical innovation: the SEON- concept for an improved cancer therapy with magnetic nanoparticles
}

\begin{abstract}
Nanomedicine offers tremendous opportunities for the development of novel therapeutic and diagnostic tools. During the last decades, extensive knowledge was gained about stabilizing and the coating of nanoparticles, their functionalization for drug binding and drug release and possible strategies for therapies and diagnostics of different diseases. Most recently, more and more emphasis has been placed on nanotoxicology and nanosafety aspects. The section of experimental oncology and nanomedicine developed a concept for translating this knowledge into clinical application of magnetic drug targeting for the treatment of cancer and other diseases using superparamagnetic iron oxide nanoparticles. This approach includes reproducible synthesis, detailed characterization, nanotoxicological testing, evaluation in ex vivo models, preclinical animal studies and production of superparamagnetic iron oxide nanoparticles according to good manufacturing practice regulations.
\end{abstract}

Keywords: cancer $\bullet$ chemotherapy $\bullet$ drug delivery $\bullet$ imaging $\bullet$ iron oxide nanoparticles - magnetic drug targeting $\bullet$ nanomedicine $\bullet$ nanotechnology $\bullet$ oncology $\bullet$ SEON-concept

Cancer remains one of the leading causes of death in the world. In 2010, the annual number of cancer incidences in the EU reached 2.6 million, and about 1.3 million deaths of cancer were recorded [1]. The upward tendency continues, in spite of all preventive measures and therapeutic efforts of recent decades [2]. Conventional chemotherapy, being applied systemically, lacks sufficient enrichment of therapeutic agents in the tumor area and negatively affects the whole organism. Severe side-effects, such as the suppression of bone marrow, dysfunction of liver and kidneys, loss of hair, as well as nausea are several consequences of this treatment. Even ototoxicity was reported for common chemotherapeutics such as platinum-derived drugs [3]. As the effectivity of cytostatic drugs correlates with the dose cancer cells are exposed to, high doses are needed for an effective therapy. Nevertheless, chemotherapy often does not lead to a complete tumor remission or is discontinued due to the severity of adverse effects [4]. Therefore, one of the future therapeutic challenges is the development of directed therapy approaches, which specifically address the tumor thus increasing the efficiency of the employed agents, without affecting the somatic tissues. Already in 1960, it was proposed that iron oxide nanoparticles in combination with magnetic fields offer a promising platform for directed drug application [5], capable of reducing the negative side-effects of conventional tumor therapy, in particular systemic chemotherapy [6] and this view has not been changed in recent years [7]. As a consequence, the diseased regions of the body are regarded as a biological unit and treated locally. Iron oxide nanoparticles represent a promising platform for targeted delivery of anticancer drugs [8] and in the last two decades, the development of nanoscaled materials to improve selective targeting has been in the scope of intense research. For the concept of magnetic drug targeting (MDT), an adequate particle size is important [9], and the nanoparticles should only be magnetized in the presence of an external magnetic field.
Stefan Lyer', Rainer Tietze', Harald Unterweger ${ }^{1}$, Jan Zaloga', Raminder Singh', Jasmin Matuszak', Marina Poettler', Ralf P Friedrich', Stephan Duerr ${ }^{1,2}$, Iwona Cicha ${ }^{1}$, Christina Janko' \& Christoph Alexiou $*, 1$ 'Department of Otorhinolaryngology, Section of Experimental Oncology \& Nanomedicine (SEON), Head \& Neck Surgery, Else Kröner-FreseniusStiftung-Professorship, University Hospital Erlangen, Glückstraße 10a, 91054 Erlangen, Germany ${ }^{2}$ Department of Otorhinolaryngology, Section of Phoniatrics \& Pediatric Audiology, Head \& Neck Surgery, University Hospital Erlangen, Bohlenplatz 21, 91054 Erlangen, Germany *Author for correspondence: C.Alexiou@web.de 
From a practical point of view, the size of superparamagnetic iron oxide nanoparticles (SPIONs) should be lower than $200 \mathrm{~nm}$ for sterile filtration, which is the easiest way of achieving sterile nanoparticles, if there are adequate filter membranes available. Additionally, the magnetic properties set some size limitations. On one hand, the maximal magnetization is falling with the diameter of the magnetic core, which is setting a lower size limit for nanoparticles that are aimed to be accumulated by magnetic fields. On the other hand, magnetic nanoparticles or agglomerates that have a size of few hundred manometers lose their superparamagnetic behavior, which could lead to thrombotic events in the target region or even in other body compartments. A common problem of many SPIONs has been that they are rapidly recognized by the reticuloendothelial system and undergo clearance, for example, via liver and spleen. This is a problem for MDT especially after systemic (intravenous, iv.) application [10]. Regarding the therapeutic applications, drug transportation in the nanoparticle-bound form makes even less soluble or instable agents available to reach tumor cells [11-13]. For a multitude of different antitumor drugs, radiotherapeutic nuclides, genetic material and antibodies, the nanoparticle delivery platform can be utilized for an improved localized enrichment [14]. Thus, drugs with highly effective cytotoxic potential, which have not yet been applicable due to their high systemic toxicity or because of metabolic barriers, can now be encapsulated in nanoparticles and applied in vivo. For oncology purposes, this means a maximized level of antineoplastic agents in the tumor area and their minimized level in the remaining parts of the body. Materials frequently used for nanoscaled solid or nanoshell based drug-delivery systems are natural or synthetic polymers, as well as metals or metal oxides. SPIONs consist of iron oxide core, often coated with organic materials such as fatty acids, polysaccharides or polymers to improve colloidal stability and to prevent separation into particles and carrier medium [15,16]. For diagnostic purposes, these nanoparticles are used for magnetic cell separation, whereby antibodies featuring a specific affinity are attached to particle surfaces. Hence, cells (e.g., circulating tumor cells from blood samples), DNA or bacteria can be separated magnetically, which for example was reported to be useful for verifying the therapeutic effect after chemotherapeutic treatment [17]. Colloidal magnetic iron oxide nanoparticles (ferrofluid) have also been implemented as contrast agents for MRI [18,19]. Currently, the marketing and the clinical application of the iron oxidecontaining contrast agents are not intensively pursued due to economic considerations of pharmaceutical companies. Commercially available contrast media such as Resovist ${ }^{\circledR}$, Endorem ${ }^{\circledR}$, Sinerem ${ }^{\circledR}$ and Combi$\mathrm{dex}^{\circledR}$ have been withdrawn from the markets, with the exception of the oral iron oxide contrast agent, ferumoxsil (Lumirem/Gastromark ${ }^{\circledR}$ ) and ferumoxytol (Rienso/Feraheme ${ }^{\circledR}$ ), a new iv. agent approved for iron replacement therapy in chronic renal failure patients with iron-deficiency anemia and experimentally used for imaging pancreatic inflammation [20]. Very early, it became clear that the magnetic properties of SPIONs allow their accumulation by means of external magnetic fields [21], as well as their application for hyperthermia therapy [22]. As demonstrated by the up to now worldwide largest in vivo study from our group, MDT using mitoxantrone-carrying SPION results in increased drug payloads in the target tissue, at the same time reducing their systemic dose and toxicity, and leading to complete tumor remissions without side-effects in a high proportion of treated animals $[23,24]$. In this work we present our understanding of the development of therapeutic SPIONs and the therapeutic strategy of MDT dedicated to the treatment of cancer and how we intend to translate this into clinical use. This includes the development and synthesis of SPIONs with a high stability in blood, their physicochemical characterization, in vitro investigations of the biological outcome including possible nanotoxicological effects, preclinical evaluation in animal experiments as well as the development of an appropriate clinical application environment and the transfer to a good manufacturing practice (GMP) - compliant manufacturing (Figure 1). Finally, we discuss the future of SPIONs and the possible position of MDT in medicine and especially oncology.

\section{Magnetic drug targeting}

The central idea of an innovative and improved cancer treatment endeavored by the section of experimental oncology and nanomedicine (SEON) is MDT. In this therapeutic concept, which was first proposed in 1960 by Freeman et al. [5], targeting is not achieved by nanoparticles coupled to molecules such as antibodies or other ligands recognizing target structures on the tumor cells, but by the physical force of an external magnetic field. Drug loaded SPIONs are applied to the vascular system and accumulated by an external magnetic field in the tumor region $[25,26]$. This strategy was already mentioned in the literature in the late 70 s and early 80 s of the last century, where Widder et al. used magnetic microspheres loaded with doxorubicin for the treatment of sarcoma bearing rats [27,28]. In 1996, Lubbe et al. used iron oxide nanoclusters stabilized with a carbohydrate coating to prevent sedimentation. Loaded with epirubicin, these particles were intravenously applied in a preclinical [29], and a first pioneer- 


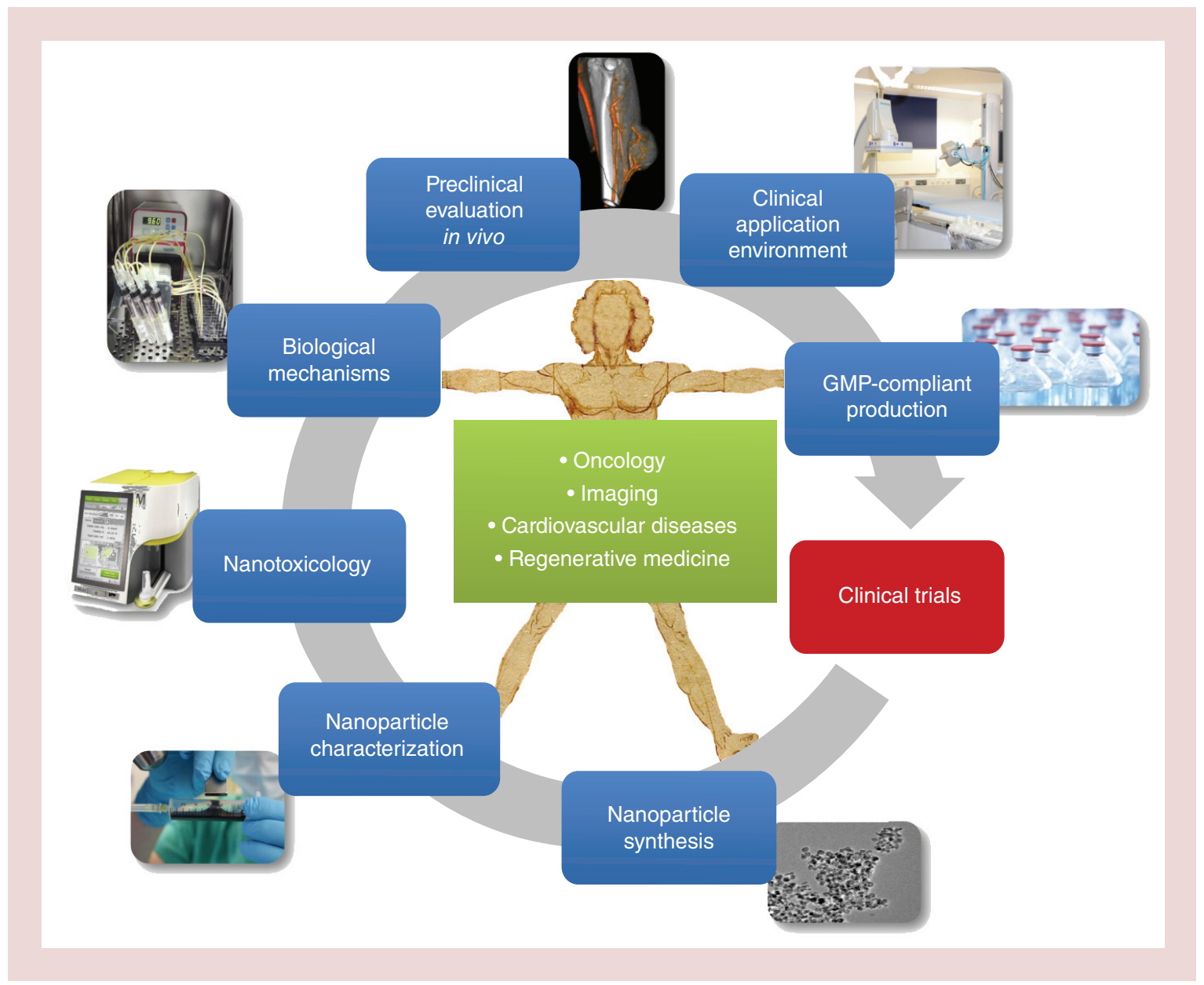

Figure 1. The section of experimental oncology and nanomedicine-concept. Section of Experimental Oncology and Nanomedicine (SEON) has been active in field of cancer therapy since 1999. The aim of SEON is to bring magnetic drug targeting (MDT) into the clinics as an enduring alternative for classical therapeutic regimes for cancer treatment. Of central importance is the development of reproducible superparamagnetic iron oxide nanoparticle synthesis suitable for GMP-conform production that can be scaled up to industry levels. To achieve this, the particles have to be evaluated as much as possible by physicochemical, in vitro and in vivo methods for important features such as stability, drug load and release, magnetic properties, toxicity or biocompatibility, effectivity and many more. But for a successful new treatment such as MDT, a suitable clinical application environment is also crucial. This includes the development of electromagnets that can be implemented into a radiological intervention suite and the evaluation of visualization methods for superparamagnetic iron oxide nanoparticles in the tumor area after the application procedure. Therefore, the SEON-labs comprise a chemical subunit for iron oxide nanoparticle synthesis and characterization, a preclinical biological subunit dealing with nanotoxicological issues as well as the biophysical mechanisms of MDT and developing applicable methods for the clinical practice of MDT. Finally, SEON has built up a GMP-facility in the pharmaceutical department of the University Hospital Erlangen.

GMP: Good manufacturing practice.

ing clinical Phase I trial with superficial tumors of different origin [30]. For nanoparticle accumulation, the authors used strong permanent magnets placed directly on the skin above the tumor. The authors concluded, that MDT with the epirubicine-loaded nanoparticles was safe and that the future potential would depend on the improvement of the nanoparticles, as well as the magnetic field used for the accumulation [30].

Taking these findings into consideration, and in contrast to the majority of other site-directed or site- specific treatment strategies developed by other groups, SEON is following an intra-arterial administration route, applying the nanoparticles near the tumor in its supporting vessels to increase the accumulation efficiency. Additionally, SEON is favoring strong electromagnets for providing the necessary magnetic field (Figure 2) [31,32].

In the following chapters we describe, how SEON intends to meet the requirements that were elucidated in the years following this first period of research in 


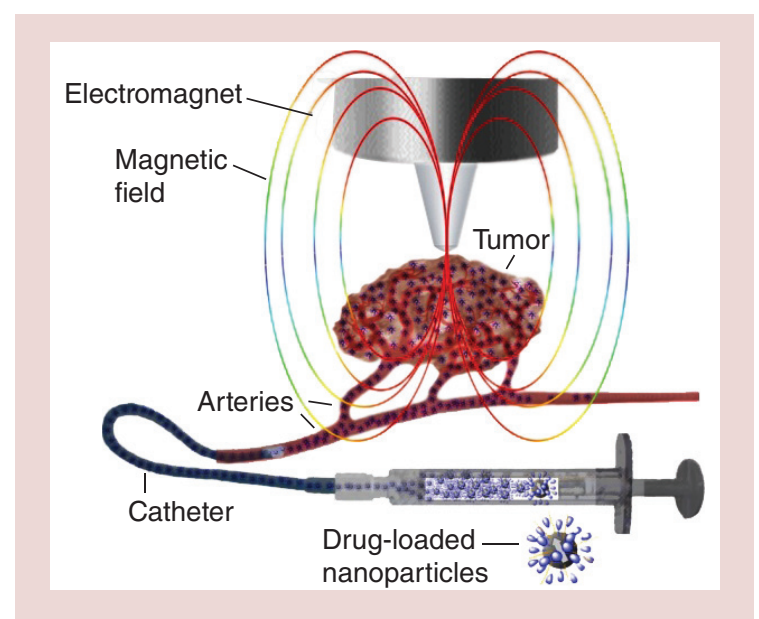

Figure 2. Magnetic drug targeting for cancer therapy. Biocompatible, stabilized superparamagnetic iron oxide nanoparticles are loaded with a chemotherapeutic drug, for example, mitoxantrone. The drug-loaded superparamagnetic iron oxide nanoparticles are administered intra-arterially via a catheter in the vicinity of a solid tumor into the vasculature supplying it, while a strong magnetic field is placed over the tumor area. Due to the magnetic force, the superparamagnetic iron oxide nanoparticles accumulate in the tumor area leading to a local drug dose, which is a multitude of what can be reached by commonly used intravenous chemotherapy. Simultaneously, the chemotherapeutic burden of the somatic tissue is substantially reduced.

the field of MDT with the clear goal of bringing this therapeutic concept into clinical use.

\section{Requirements for clinical studies}

Numerous regulatory requirements have to be fulfilled if a medicinal product or drug is intended for human application [33,34]. To name them all in detail would exceed the scope of this article. However, it should be noted that the regulatory agencies established very precise and well-defined benchmarks for characterization, which have to be addressed before applying for a clinical study. These benchmarks involve requirements for the synthesis, for example concerning purity, batch-tobatch conformity, analytical methods thereof, absence of pyrogens, sterility, stability and last but not least a well-documented synthesis procedure which follows GMP guidelines. Concerning the biological part, detailed toxicological assessments following good laboratory practice (GLP) must be performed, including the evaluation of the short- and long-term toxicity/biocompatibility of those particles for an appropriate risk assessment. Finally, the product has to prove its efficacy and safety in two different animal species, at least one of which being nonrodent. All these characterizations merge in the so-called investigational medicinal product dossier (IMPD), which contains all relevant scientific information and has to be submitted together with the application for approval.

However, addressing these standard procedures may not be enough to successfully translate nanoparticle formulations into clinical studies. Many analytical standard methods that are frequently used in physicochemical and biological characterization of small molecule drugs may not be suitable to sufficiently characterize nanoparticle formulations, whereas other, innovative approaches may be better suitable, but are not well established in clinical routine analysis. In this respect, the EMA created the Innovation Task Force (ITF) in 2006 to deal with the challenge of standardization of methods for novel medicinal products [34]. This process is currently still ongoing, and the methods and requirements have not been completely regulated so far [35]. For new nanoparticle therapy approaches, this means that alternative concepts may have to be found individually in order to demonstrate conformity of the nanoparticle system with EMEA or US FDA guidelines.

The SEON-concept has emerged from this requirement and incorporates all the different aspects of translational nanomedicine, from research and development to GMP synthesis. In the following paragraphs we describe our approach in respect to current scientific standards.

\section{Iron oxide nanoparticle synthesis \& coating}

Multiple techniques for the synthesis of SPIONs have been described so far [36]. For biomedical applications, coprecipitation and thermal decomposition have evolved as the most common methods [6]. Coprecipitation involves the formation of magnetic iron oxides (either $\mathrm{Fe}_{3} \mathrm{O}_{4}$ or $\gamma-\mathrm{Fe}_{2} \mathrm{O}_{3}$ ) from a solution of ferric and ferrous salts by addition of a base. This method is easy to carry out and does not involve organic solvents. It consists of only one main step and is therefore easy to control, enabling up-scaled synthesis with relatively low effort. However, allows only a poor control over shape and size distribution of iron oxide cores. Thermal decomposition is usually performed on organic iron precursors such as $\mathrm{Fe}(\mathrm{acac})_{3}$ or $\mathrm{Fe}(\mathrm{CO})_{5}$. At high temperatures $\left(>100^{\circ} \mathrm{C}\right)$, the organic compound is decomposed and nanocrystalline iron oxide is formed. Choice of appropriate temperatures and reaction times enables tight control over shape, size and size distribution of such particles [37]. Obviously, these reactions are usually performed in organic high-boiling solvents and for medical use the particles have to undergo a phase transfer into aqueous media $[38,39]$. These procedures can be easily done in laboratory scale. However, they involve an additional volume-sensitive step, which could cause problems when the method has to be up-scaled. 
Clinical nanoparticle formulations need an upscaled, reproducible synthesis pathway. Toxic synthesis residuals are to be avoided.

Therefore, coprecipitation seems to be the better choice for SPIONs dedicated for MDT and consequently is used at SEON. However, this may not necessarily apply to all biomedical applications and a choice has to be made individually, taking the respective requirements into account.

While the fundamental synthesis methods of iron oxide nanoparticles did not change much in recent years, the area where most research has been performed is the modification of particle coatings $[6,8]$. The reason is obvious: The surface of nanoparticles defines many of their reactions at the bio-nano interface [40], namely cellular uptake, hemocompatibility, toxicity and colloidal stability.

The most frequent coating substances include complexing agents, fatty acids, polysaccharides, other biocompatible polymers and proteins [6]. All these substances are able to produce core-shell SPION formulations which are colloidally stable and nontoxic in vitro. However, in recent times, the complexity of the reactions at the bio-nano interface in vivo is coming into focus more and more. Upon injection into blood vessels, many nanoparticles are almost immediately surrounded by a protein corona, which consists of a mixture of plasma proteins [41]. This corona can contribute to colloidal stability, but also greatly affect cellular uptake of particles and, consequently, their biocompatibility [42]. Moreover, it can promote the elimination of the particles from the circulating system by macrophages. However, upon intra-arterial MDT the latter effect is not necessarily relevant, as the particles do not enter central circulation before reaching the tumor.

Developing own SPIONs or nanoparticles guarantees a shorter and more direct feedback loop between nanoparticle synthesis and biological characterizations, which in our opinion is crucial for a successful translation of the whole concept into a clinically relevant application.

To date, SEON has developed two main platforms (Figure 3) capable of binding a variety of different drugs for different purposes. One of them, SEON ${ }^{D E X}$, is represented by SPIONs stabilized by a cross-linked dextran coating and able to bind drugs via an additional functionalisation with hyaluronic acid [43]. The synthesis is modified after Kamat et al. [44] starting with coprecipitation of SPIONs in a mixture dextran solution. After purification, the dextran coating is crosslinked using epichlorohydrin. After this step, the dextran is functionalised with hyaluronic acid and loaded with the cytostatic drug cisplatin.
The resulting cisplatin-containing functional particles (SEON ${ }^{\text {DEX-HA*CPt }}$ ) are nanoclusters of about $75 \mathrm{~nm}$, which is about three-times the size of the basic system SEON ${ }^{\mathrm{DEX}}$. The size of the single cores is approximately $4 \mathrm{~nm}$.

The second evaluated SPION system (SEON ${ }^{\mathrm{LA}-\mathrm{BSA}}$ ) is stabilized by a lauric acid coating and an additional artificial protein corona consisting of serum albumin [45]. The synthesis is modified after Bica et al. [46] starting with an alkaline precipitation of nanoparticles at $80^{\circ} \mathrm{C}$. After coprecipitation, the particles are colloidally stabilized using lauric acid. The resulting ferrofluid SEON ${ }^{\mathrm{LA}}$ is dialyzed and added dropwise to a serum albumin solution for final coating. After several filtration steps, a chemotherapeutic agent can be bound to this system. The loading capacity of e.g. mitoxantrone is up to $300 \mu \mathrm{g} / \mathrm{ml}$ without changing the final size of the nanoparticles of about $60 \mathrm{~nm}$. Using this method, the single cores usually have a diameter of about $7.6 \mathrm{~nm}$.

These two examples show, how different the routes of nanoparticle synthesis can be, nevertheless leading to functional nanoclusters that can and have to be analyzed further, before they can be used in vivo.

Both basic systems are serving as nanoparticulate platform technology for a big variety of different drugs as well as therapeutic and diagnostic fields of operation.

\section{Physicochemical characterization}

Detailed characterization of SPIONs is not only of outstanding importance for an explicit understanding of the particles, but is also crucial for establishing a reproducible synthesis. This is valid for all kinds of nanoparticles, especially if they are intended for therapeutic and/or diagnostic use in humans and animals [6,47].

Due to being composed out of different materials and their complex physical and chemical properties, a whole panel of techniques covering chemistry, physics, biochemistry, biomedicine and molecular biology has to be utilized for nanoparticle characterization. Physicochemical characterization of SPIONs allows determining the correlation between synthesis parameters and nanomaterial properties. Large surface area and small size of nanoparticles provide properties distinct from those of bulk materials. The surface properties of SPIONs strongly influence their aggregation behavior in biorelevant fluids, like cell culture media, isotonic salt solutions, blood, the cytoplasm of cells or in cell suspensions, which consequently influences their migration behavior, uptake and toxicity [48]. Various physicochemical characteristics of particles such as crystallinity, magnetization, particle size and size distribution, composition of surface coatings, aggregation status in the relevant media and the purity of the sam- 


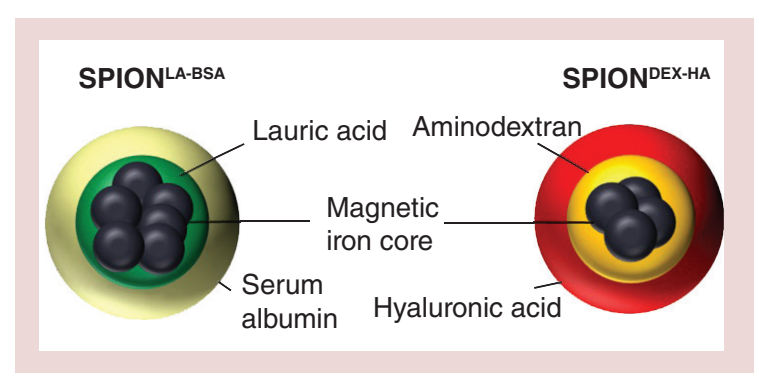

Figure 3. Schematic presentation of the current two basic nanoparticle system synthesized by section of experimental oncology and nanomedicine. Both superparamagnetic iron oxide nanoparticle-systems have a core of nanoclusters surrounded by a stabilizing and biocompatible shell, which additionally is the basis for the functionalization with chemotherapeutics of other drugs or biologicals.

DEX: Dextran; HA: Hyaluronic acid;

SPION: Superparamagnetic iron oxide nanoparticle.

ple have to be evaluated to avoid side-effects before any in vitro and in vivo tests.

Using contemporary characterization methods, including determination of nanoparticle morphology (transmission electron microscopy), hydrodynamic size and zeta potential (dynamic light scattering), chemical composition (infrared spectroscopy) and magnetic properties by magnetization analysis [49,50] is necessary to receive a comprehensive picture of the particle properties, which is shown in Figure 4 as an example for SEON ${ }^{\text {LA-BSA }}$ nanoparticles. A very useful method for analyzing surfaces is the $\mathrm{pH}$ dependent or ionic strength dependent measurement of hydrodynamic size and surface charge by dynamic light scattering [51]. This method offers a comprehensive characterization of particle surfaces and, consequently, an estimation of colloidal stability of SPIONs in biological fluids. More sophisticated spectroscopic in-depth investigations of the chemical composition of the coating shells of SEON nanoparticles are currently done in cooperation with other research groups by $\mathrm{x}$-ray photoelectron spectroscopy or small angle scattering. The quality of modern complementary small angle $\mathrm{x}$-ray scattering and small angle neutron scattering allows comprehensive characterization of particle structures. It may even be possible to locate certain molecules such as drugs in the particle structure [52].

It is obvious that the magnetic properties of SPIONs are one of the most important parameters for their feasibility for MDT. The magnetic profile of such particles is strongly related to the accumulation efficiency during MDT: The higher the saturation magnetization of the ferrofluid, the easier the particles can be accumulated with a magnetic field. On the other hand, irreversible clustering of the particles under the influence of a magnetic field, or even by remnant magnetization raises the danger of thrombotic events during MDT therapy. Therefore, high colloidal stability of SPIONs, even in complex media and under the influence of a magnetic field, is of utmost importance.

In cooperation with partners the magnetic properties of SPIONs produced at SEON are measured by vibrating sample magnetometry [53] and magnetorelaxometry (MRX) [54]. Additionally, with MRX it is possible to measure the content of iron oxide nanoparticles independent of natural, nonmagnetic iron in biological tissue [55,56] or even in vivo in rabbits [57] or mice [58].

Finally, SEON keeps a well-defined galenic profile of their nanoparticle systems. Parameters such as drugloading capacity and the influence of drug conjugation on other parameters of the nanoparticles (namely their size and colloidal stability) have to be well characterized. The drug release profile in various media (e.g., cell culture media, blood) is of further importance, as the particles remain in the targeted tissue after accumulation [23]. For approval as medical drug, those pharmacokinetic parameters need to be thoroughly investigated [59]. Another very important aspect that is often overlooked at the research and development stage is the stability of particles during storage. Just recently a pilot study showed encouraging results, but also raised interesting questions [60]. SEON ${ }^{\mathrm{LA}-\mathrm{BSA} A}$ particles were stored over 12 weeks at different temperatures ranging from 4 to $45^{\circ} \mathrm{C}$. Over this time range, alterations in color, crystal structure and saturation magnetization, as well as cellular uptake and toxicity were detected. On the other hand, colloidal stability and chemical composition were not affected even after 12 weeks of storage at $45^{\circ} \mathrm{C}$. These findings raise the question whether the storage conditions and the respective alterations have any relevant influence on the in vitro and in vivo efficiency of such systems.

\section{Biophysical interplay of SPIONs \& the magnetic field}

The interplay between magnetic nanoparticles, magnetic forces and other forces such as hydrodynamic drag forces is influencing targeting efficiency and therefore it is important to study those effects.

The Basis of in silico simulations of such dynamic interactions usually are tube models with or without branches and the common result is that the targeting efficiency is rising with increasing magnetic forces, declining vessel diameters and falling blood velocity [61]. But in those simulations, the magnetic drag forces are too small for an efficient targeting [62].

Nevertheless, there are in vitro models available, which attempt to match experimental data with simulations and showed that it is possible to accumulate nanoparticles in a tube against the drag forces of a 
flow $[63,64]$. Another study demonstrated that it is dependent on the position and strength of the magnetic field how much of a ferrofluid can be directed into a side branch of a bifurcation tube system [65].

SEON utilizes two different models for experimental MDT simulation under flow conditions. We developed an ex vivo model based on bovine femoral arteries [66] for studying the effects of different parameters, such as flow velocity, magnetic field strength and different distances between the pole shoe and the target site. In this circulation model, it was demonstrated, that it is possible to accumulate magnetic nanoparticles under the tip of the poleshoe of an electromagnet against the drag forces of a flowing buffer. This was shown qualitatively by staining of iron oxide nanoparticles with Prussian blue in histological cross sections and micro computed tomography $(\mu \mathrm{CT})$ and quantitatively by magnetrelaxometry [66-69]. Currently, we are working on a bigger set of experiments completing these preliminary studies.

The second model is an in vitro model of arterial bifurcations, originally developed for flow experiments in atherosclerosis research [70]. In this model, we already showed, that it is possible to magnetically accumulate SPIONs at the bifurcation and that the nanoparticles are taken up by human umbilical vein cells (HUVECs) in this region [71].

Taken together, the current available in silico models, showing that magnetic accumulation of nanoparticles flowing nanoparticles is not possible, may lack some critical information, for example, about magnetic interactions between the nanoparticles. However, such

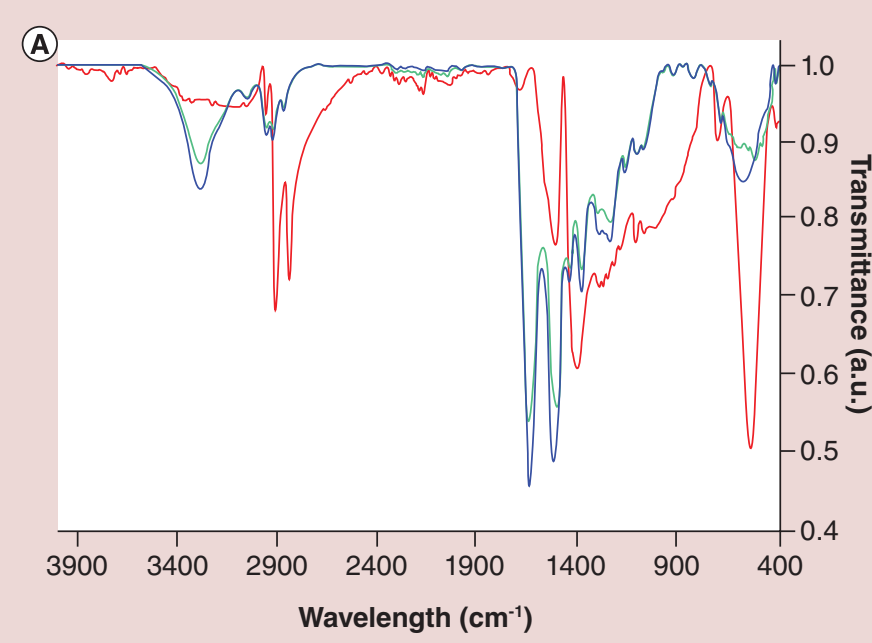

(B)
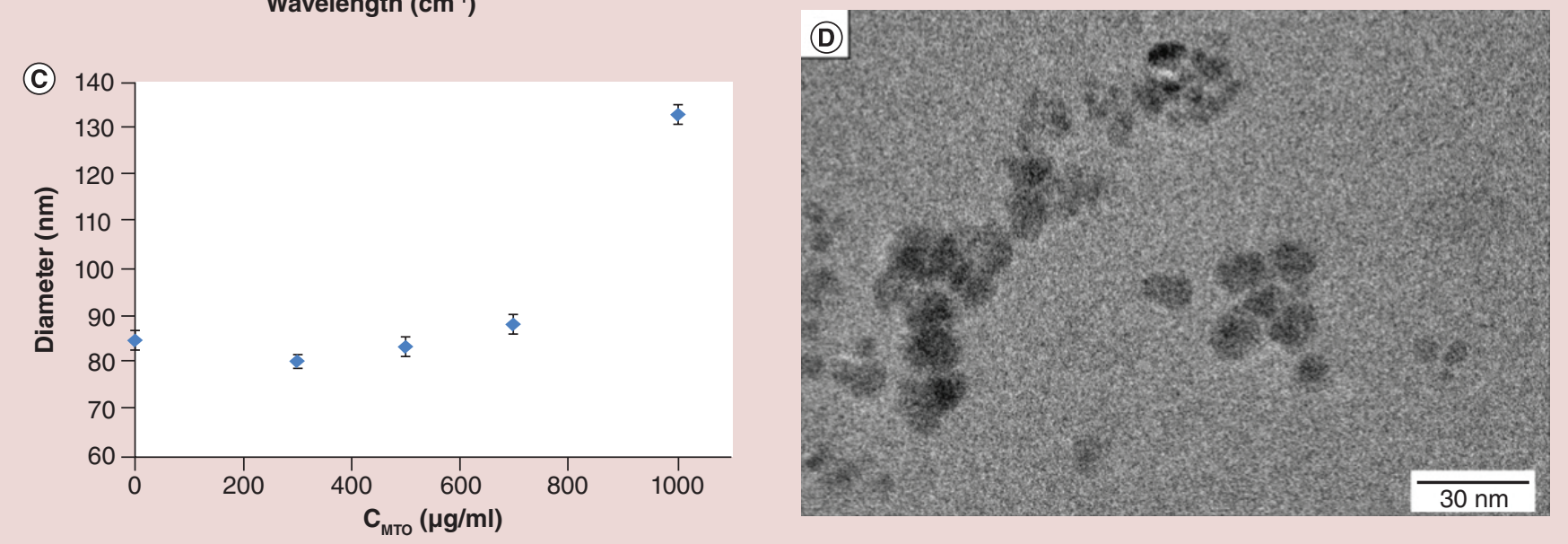

Figure 4. Superparamagnetic iron oxide nanoparticle characterization results for lauric acid- and bovine serum albumin-coated superparamagnetic iron oxide nanoparticles (SEON ${ }^{\mathrm{LA}-\mathrm{BSA}}$ ). (A) Infrared spectroscopy measures the subsequent surface modification. The spectra of the lauric acid coated superparamagnetic iron oxide nanoparticles (SEON ${ }^{L A}$ ) in red can be clearly distinguished from those of lauric acid-bovine serum albumin hybrid layers (SEONLA-BSA; blue line). The BSA spectrum was inserted for comparison (green line). (B) Hydrodynamic diameter measured by dynamic light scattering. The mean diameter ranges between 50 and $60 \mathrm{~nm}$. (C) Influence of mitoxantrone loading to SEON ${ }^{L A-B S A}$ on the particle size measured by dynamic light scattering. (D) Corresponding transmission electron microscopy pictures of SEON ${ }^{L A-B S A}$ nanoparticles.

SEON: Section of Experimental Oncology and Nanomedicine. 
Perspective Lyer, Tietze, Unterweger et al.

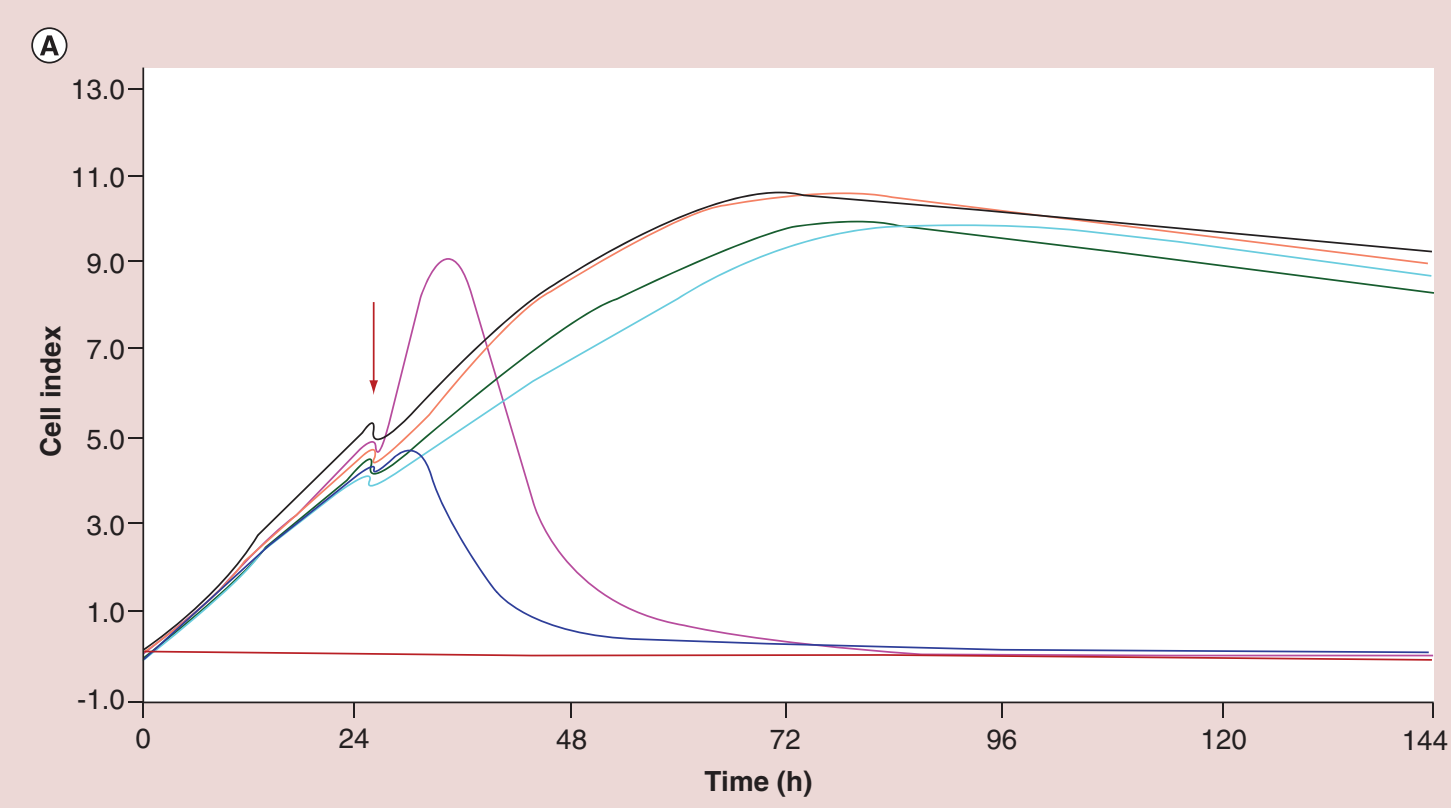

(B)

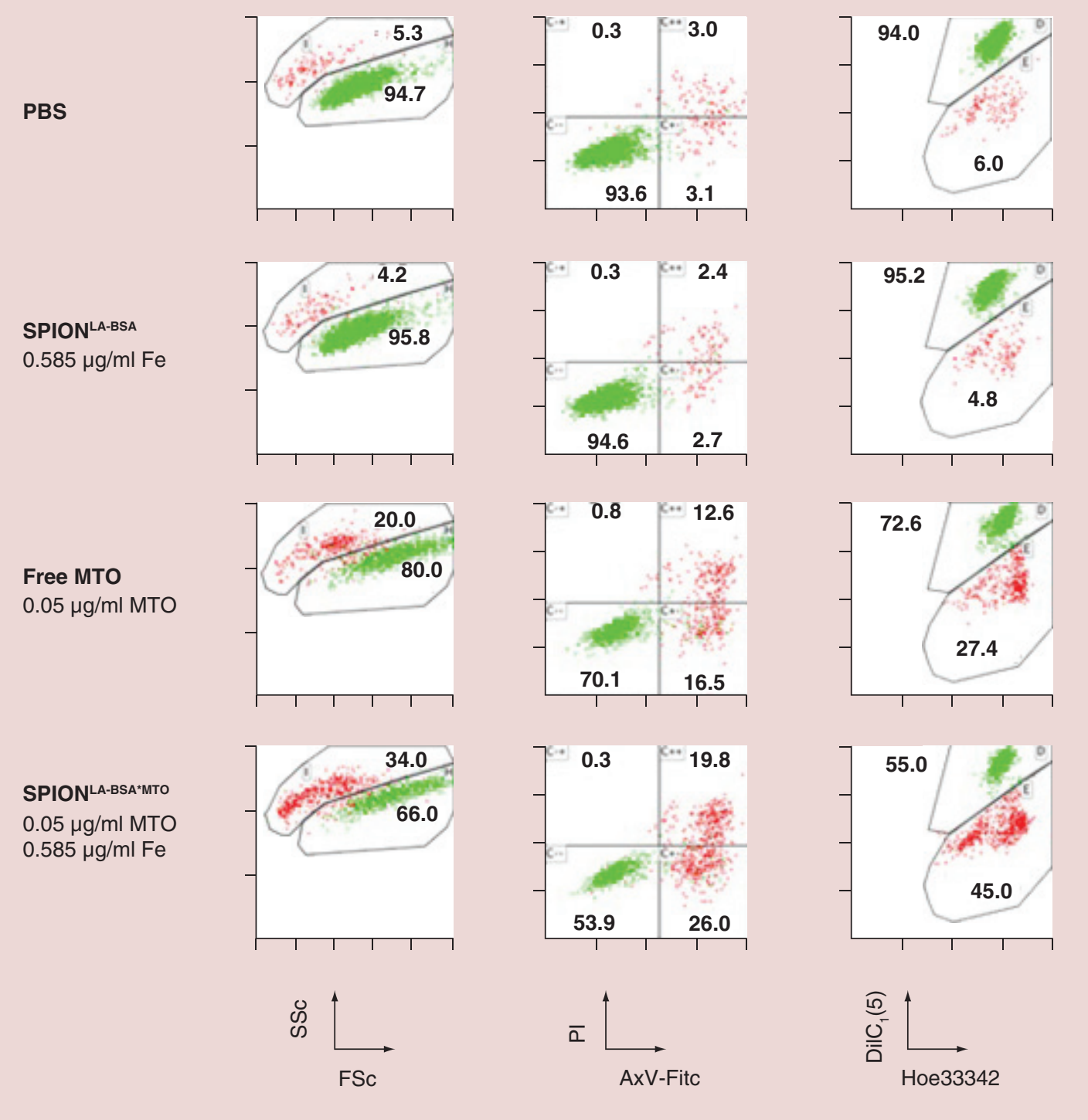


Figure 5. Combination of complementary methods enables the valid analysis of nanoparticle mediated effects in vitro (see facing page). (A) Real-time cell analysis based on impedance measurement (xCELLigence ${ }^{\oplus}$ technology) enables the label-free monitoring of adherent cells for several days. Viable proliferating adherent cells are usually tightly attached to plastic surfaces and cause impedance, which is indicated by an increasing cell index. Dying cells in contrast detach, which is reflected by decreasing cell indices. Here shown: RTCA-evaluation of pure SPIONs over a period of $144 \mathrm{~h}$ in the breast cancer cell line MCF-7. Red curve: pure cell medium as baseline; green curve: cells without test substance; orange curve: $0.7 \mu \mathrm{g} / \mathrm{ml} \mathrm{SPIONs;} \mathrm{black} \mathrm{curve:} 7 \mu \mathrm{g} / \mathrm{ml}$ SPIONs; light blue curve: $70 \mu \mathrm{g} / \mathrm{ml} \mathrm{SPIONs;} \mathrm{pink} \mathrm{curve:} 700 \mu \mathrm{g} / \mathrm{ml} \mathrm{SPIONs;} \mathrm{dark} \mathrm{blue} \mathrm{curve:} 1400 \mu \mathrm{g} / \mathrm{ml}$ SPIONs; red arrow: substance addition. (B) Flow cytometry analyzes of the effect of SEON ${ }^{L A-B S A}$, SEON ${ }^{L A-B S A}$ loaded with MTO and free MTO without nanoparticles. (B) Flow cytometry analyzes of the effect of SEON ${ }^{L A-B S A}$, SEON ${ }^{\text {LA-BSA }}$ loaded with MTO and free MTO without nanoparticles. (B) Multiparameter stainings in flow cytometry provide a comprehensive picture on induced cell death phenotypes. Annexin-Fitc (AxV-Fitc) binds to hosphatidylserine, which is marker for apoptosis; PI intercalates into the DNA of cells with disturbed plasma membranes, being a marker for necrosis; DilC1(5) provides information about the mitochondrial membrane potential (viable cells are positive for DilC1[5]) and Hoechst 33342 is a plasma membrane permeable dye that supports the discrimination between cells and nanoparticles for analysis. Due to the intrinsic fluorescence of MTO, additionally the intracellular amount of the cytotoxic drug can be estimated. Viable cells are depicted in green; apoptotic/necrotic cells are depicted in red. BSA: Bovine serum albumin; LA: Lauric acid; MTO: Mitoxantrone; PI: Propidium iodide; SEON: Section of Experimental Oncology and Nanomedicine; SPION: superparamagnetic iron oxide nanoparticle.

(A) Reproduced with permission from [83].

simulations probably closely describe the situation in which intravenously applied SPIONs with a short blood half-life and a low particle concentration pass the magnetic field. These conditions are significantly different from the intra-arterial approach we are favoring. A cross-talk between scientists performing in silico simulations and scientists producing experimental data could improve the mathematical predictions and further help to optimize magnet positioning and particle accumulation, both in preclinical and clinical settings.

\section{Biological characterization \& nanotoxicology/biocompatibility}

The development of nanoparticles dedicated for medical use has to be accompanied by nanotoxicological or, more positively named, biocompatibility testing. Thus, this area of research has been gaining more and more importance in the field of nanotechnology in the recent years [72,73].

However, searching the current literature, one encounters some problems of nanotoxicological studies. Today, nanoparticles are often developed in excellent chemical laboratories, but biologically analyzed in other working groups regarding in vitro and in vivo effects. This constellation includes an intrinsic risk of loss of information between these two laboratories. This can lead to misunderstandings or misinterpretations of results [74,75]. On the other hand, a large number of toxicological studies are performed with commercially available nanoparticles, lacking an appropriate amount of information about the physicochemical properties.

Therefore, many existing nanotoxicological studies are not suitable for risk assessment, because of the missing description of essential particle characterization as well as unclear indications of concentrations, or contain methodical uncertainties [76,77].
Another repeatedly neglected problem is that many nanoparticle types interfere with commercially available toxicity tests. Especially iron oxide nanoparticles represent a major problem for such assays, since the nanoparticles are optically disturbing the absorbance, luminescence or fluorescence signals. Therefore, classical absorption assays as, for example, MTT are not applicable for the analysis of particular nanoparticles and have been noted to deliver a wide range of false positive as well as false negative results [73,78-80]. Consequently, the toxicity of nanoparticles as described in the literature often should be questioned, or reinvestigated.

Hence, the SEON-concept comprises the combination of chemical expertise in developing and synthesizing/characterizing iron oxide nanoparticles with biomedical expertise performing the preclinical in vitro and in vivo testing. We believe that only in such a combination it is possible to achieve an appropriate picture of biological and toxicological effects of specific nanoparticles.

Furthermore, at SEON a combination of assays and methods differing from the classical ones is used. SEON is currently working on a standardized workflow for the toxicological testing of nanoparticles including real time cell analysis techniques, like real time cell analysis (RTCA) with the xCELLigence ${ }^{\circledR}$-system, flow cytometry (FC) assays as well as life cell and fluorescence microscopy (IncuCyte ${ }^{\circledR}$, ZEISS Observer ${ }^{\circledR}$ ). RTCA is a very sensitive technique based on impedance measurements in well-format suitable for testing adherent cells [81]. In this system toxicology (Figure 5A) as well as efficiency studies of nanoparticles loaded with chemotherapeutic drugs can be performed [82,83]. Flow cytometry has been used in biomedical research for a long time and has some advantages, when utilized for nanotoxicological testing of nanoparticles. One of the biggest advantages is that the nanoparticles remain- 
ing in the solution do not disturb the measurement, because they can be easily discriminated from the cells by means of their size [84]. In addition, a multiplexing of assays is possible with FC (Figure 5B). In a multicolor assay apoptosis, necrosis, mitochondrial membrane potential and DNA-degradation can be monitored simultaneously in a high-content approach. Moreover, based on the granularity of the cells, SEON has developed a method for measuring the SPION content within cells, a parameter which is usually neglected in respective studies [85].

We are strongly convinced, that just by merging these techniques it is possible to draw a realistic picture of the effects nanoparticles have on cells in vitro and that this finally has to be completed by appropriate in vivo tests.

\section{In vivo application of MDT}

A number of working groups worldwide are developing and testing magnetic nanoparticles for therapeutic and diagnostic purposes $[6,26]$. Several groups already have been using in vivo models, albeit aiming at different goals. In the field of tumor diagnosis for example, SPIONs are utilized for detecting malignant lymph nodes with MRI [19], as well as tracking SPION labeled stem cells with MRI [86] or ultrasound [87].

The first animal trials found in literature were performed in rats $[28,88]$. Since then SPION studies have been carried out in rats, mice, rabbits, guinea pigs, hamsters and pigs. Apart from some exceptions, the route of application usually is intravenous injection, which in our opinion has at least two main disadvantages. First of all, the dilution of the particles in the circulation after intravenous application dramatically reduces the efficiency of magnetic targeting. Second, the blood half-life of nanoparticles is usually quite short and most of the SPIONs are removed from the circulation during the first pass through the liver $[6,36]$.

Therefore, the concept for MDT favored by SEON is an intra-arterial approach, which admittedly needs more effort during the application procedure, and is furthermore difficult to perform in mouse or rat as model organisms. Hence, we chose the rabbit model of VX2, which is a squamous cell carcinoma originating from a rabbit papilloma [89,90]. This tumor is transplantable in rabbits to different body regions or organs [91-93], and for the SEON-model is routinely implanted subcutaneously at the hind limb of New Zealand White rabbits [31,94]. The subsequent MDT treatment is performed by an intra-arterial access and nanoparticle administration through the femoral artery in the proximity of the tumor, while a strong electromagnet is placed at the tumor surface. The great potential of MDT was shown already in 2000 by Alex- iou et al. albeit with a limited animal number [31]. In the following years the in vivo behavior of the nanoparticles was studied. The enrichment of nanoparticles in the tumor and tumor region was demonstrated in several studies and by different techniques [9,55,95-99]. Together with colleagues from the 'Physikalisch Technische Bundesanstalt Berlin' (PTB) and from the Technical University in Dresden, nanoparticle accumulation in tumor tissue could be measured quantitatively with MRX [68], and qualitatively, as well as semiquantitatively imaged with $\mu \mathrm{CT}[100,101]$.

However, for proper pharmacokinetic and biodistribution studies, the ability to detect and quantify the administered drug is crucial. This is possible for mitoxantrone (MTO) from blood and different tissues by a quantitative HPLC protocol. But upon nanoparticlecoupling of MTO this protocol did not deliver reproducible results, especially from liver tissue. Therefore, the chemical analytics subunit of SEON had to develop a new quantification protocol for SPION-bound MTO in complex tissues [102].

Another important issue for in vivo MDT is the vascular structure, which is supplying the tumor. A detailed knowledge of these arteries is crucial for the intra-arterial approach: the arterial branches supporting the tumor must be known in order to place the magnet at the optimal position. Together with colleagues from the Department of Neuroradiology of the University Hospital of Erlangen, the C-arm cone beam computed tomography (Artis zee floor, Siemens Healthcare, Forchheim, Germany) was found suitable for displaying the respective vessels in the rabbit model (Figure 6) [94], which is also constitutes another step towards a realistic clinical setting. Currently, the next step is done by exchanging the direct femoral by an access through the carotid artery and a catheter-based application of the nanoparticles.

Finally, in 2013 SEON published the worldwide largest preclinical animal study for MDT to date, which included $67 \mathrm{New}$ Zealand white rabbits [24]. No accumulation of MTO or MTO bound to nanoparticles was observed $24 \mathrm{~h}$ after intravenous administration even under the influence of a magnetic field gradient of up to $72 \mathrm{~T} / \mathrm{m}$ measured directly at the poleshoe. Less than $1 \%$ of the recovered MTO was localized to the tumor region. By contrast, $57 \%$ of the recovered MTO was detected in the tumor area after intra-arterial MDT with the same magnetic field parameters. Consequently, the therapeutic part of the study showed a significant higher survival probability in the intraarterial MDT groups compared with the untreated controls (eight animals). Moreover, after only one single treatment with a dose of only up to $10 \%$ regular single dose of MTO the MDT-treatment group 


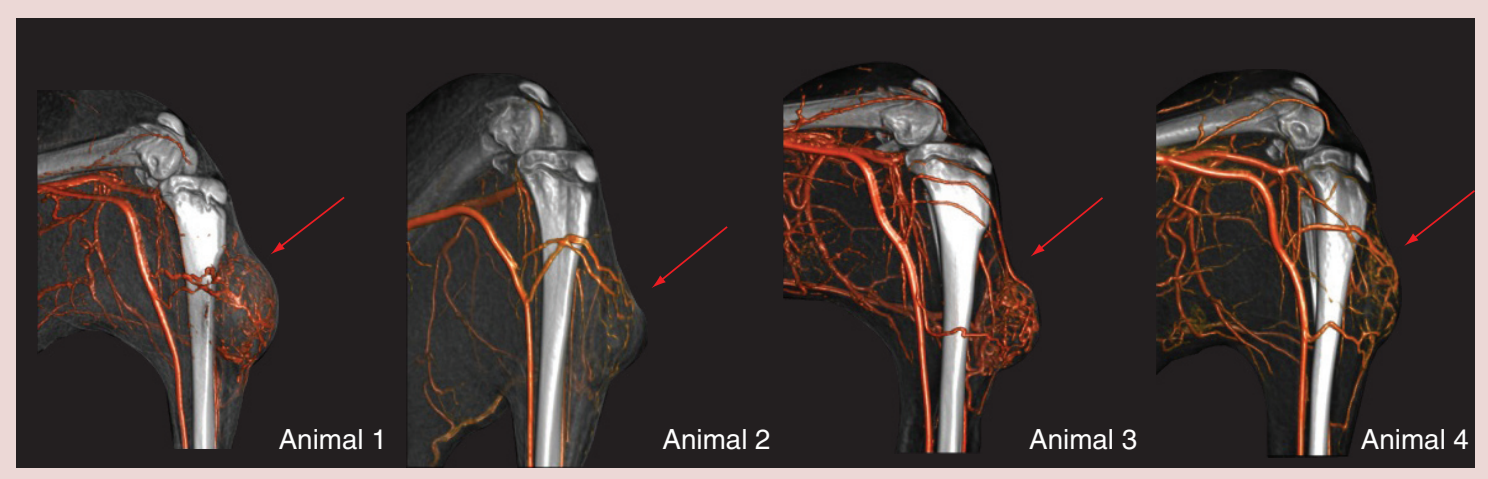

Figure 6. Angiographic images of the left hind limb of rabbits with subcutaneous implanted VX2-tumors. Angiographic presentation (3D volume rendering technique [VRT]) of four different VX2-tumors, each implanted at the left hind limb of four New Zealand white rabbits (bones and soft tissue are displayed in grey; vessels are displayed in dark orange. In all four animals, the tumor is placed lateral at the left hind limb approximately $2 \mathrm{~cm}$ below the knee. Although some similarities can be seen, the vascularization of all four tumors is very different. For an effective treatment with magnetic drug targeting an exact knowledge of the tumor vascularization is crucial for placing the magnetic field at the right position. Red arrow: tumor area.

(20 animals) ca. $30 \%$ of the tumors showed complete remissions and no recurrence or metastases during the follow-up period of 120 days (Figure 7).

\section{Conclusion}

Diseases such as cancer, atherosclerosis or severe clinical situations such as sepsis are leading causes of death worldwide and to date diagnosis and therapies despite tremendous efforts that have been put into biomedical research remain still challenging. Nanoparticulate formulations have been promising to overcome the limitations of small molecules mainly by improving the bioavailability of the drug at their destinations, in other words, the diseased area(s). Nevertheless, in nanomedicine, like in all areas of research, the single scientist in general is convinced to develop concepts to solve upcoming tasks. Since science needs public funding, especially in terms of being independent, the competition receiving support from this source is very high.Here, social sciences could act as a kind of attendant, being not involved directly in the research and development process. Social scientists could create an overview about existing research knowledge combined with the knowledge about common social coherences. This could offer the opportunity to find synergies between the different disciplines, accelerating the development of therapies and diagnostics. What would necessary for this? Based on literature and first cooperations, social scientist would have to conduct their research avoiding judgements for certain nanotechnological areas of research and such retrieved information should not influence decisions made by funding agencies. By this, the necessary confidence could be created bringing nanomedical scientist to open part of their research to social scientists. In a next step, there has to start an open and confidential discussion between the social and the nanomedical scientists about the results of 'nanosocial' science. In the the future this could create a new opportunity and scientists and patients could profit from new ideas and possible new shortcuts for bridging the difficulties of scientific knowledge to reach the clinical routine.

\section{Future perspective}

We believe in the high potential of magnetic nanoparticles as well as intra-arterial MDT for an improved cancer therapy and are following a consequent route through all fields of nanomedical and nanotechnological research that in our opinion today is reasonable and available. At SEON, the synthesis on a lab- and GMP scale and a large panel of physicochemical characterization techniques suitable for SPIONs are available. This is complemented by biological and nanotoxicological in vitro evaluations of the developed nanoparticles with direct feedback to the synthesis unit. Furthermore, a preclinical treatment facility with an operation theatre and a C-arm cone beam computed tomography for angiographic imaging allows performing the proof of feasibility and efficacy studies in animal models (Figure 8). SEON scientists are working on improving the available analytical methods and on establishing new standards for the characterization of magnetic iron oxide nanoparticles. Finally, intense efforts are focusing on the realization of a suitable clinical application environment with the respective safety matters. Since 1999, when the first experiments were done with an immovable electromagnet weighing $1.500 \mathrm{~kg}$, the current electromagnet has evolved to a movable device of about $47 \mathrm{~kg}$ with comparable magnetic field parameters [32]. Today, these magnets can reach a penetration of only a few centimeters into the tissue, which limits MDT to superficial tumors, for example, head 


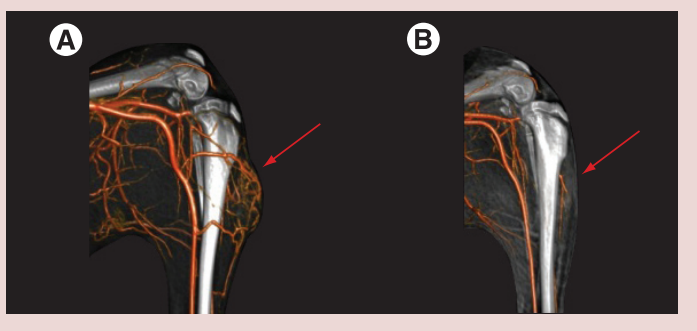

Figure 7. Angiographic image of the left hind limb of a rabbit with a VX2 tumor before and after magnetic drug targeting. Imaging was done (A) directly before magnetic drug targeting and (B) 11 weeks later. It can be seen that 11 weeks after one single treatment with $10 \%$ of a regular systemic dose, the tumor had disappeared completely and no adverse side-effects occurred. Red arrow: tumor area.

and neck cancers or sarcoma of the extremities. Therefore, much effort has to be put on magnet development and improving the magnetic field for gaining a deeper penetrance into the human body. At the same time, tumor models have to be established that mimic cancers in deeper body compartments and organs that are intended to be treated. These models have to prove, that MDT can be used for the therapy of cancers of, for example, the liver, prostate or pancreas. Additionally, these models could show, whether the high drug doses that can be achieved in the tumor region by MDT can overcome the influence of the tumor microenvironment in the respective organs. If the deeper penetrance of the magnetic fields can be achieved and MDT proofs to be effective for tumors in deeper body compartments, the

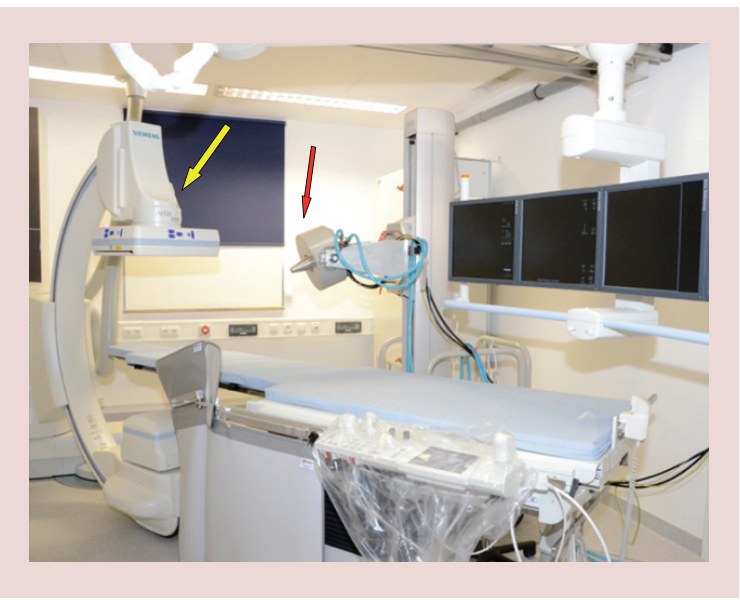

Figure 8. Preclinical operation theater at the Section Of Experimental Oncology And Nanomedicine facilities. Section of Experimental Oncology and Nanomedicine has established a preclinical operation theater with an electromagnet (red arrow) that is adjustable in all three room directions (magnetic field parameters at the tip of the poleshoe: max. field strength: $1 \mathrm{~T}$; max. field gradient: $72 \mathrm{~T} / \mathrm{m}$ ) and a c-arm cone beam CT (Artis zee floor, Siemens Healthcare, Forchheim, Erlangen) for angiographic imaging (yellow arrow). number of patients, which can benefit from MDT, will rise tremendously.

SEON has shown the effectivity of MDT for the treatment of superficial tumors utilizing lab-scale synthesized nanoparticles. This and their in vivo toxicological profile, now have to be re-evaluated with GMP-compliant nanoparticles under good laboratory praxis (GLP) - conditions, before clinical studies can be started. In these studies, which are of utmost importance for the approval of the nanoparticles for the use in humans external institutions have to be involved, which are specialized on performing the respective GLP-studies in vitro and in vivo, to fulfil the requirements of the regulatory bodies.

We believe that the potential of MDT and magnetic nanoparticles as a platform technology can be translated to other diseases such as the treatment of arteriosclerosis [103], local inflammation or local infections and that this method could be modified for applications in regenerative medicine [104].

The past has shown that the treatment of cancer with monoclonal antibodies is less effective and less selective as scientists and clinicians hoped and that there is a long way to go until the former goals may be reached. Here, SPIONs as well as other nanoparticles could be used as platform for the combination of different molecular targeting strategies with cytostatic drugs. By combining this approach with MDT, tumor targeting could be improved even further. However, it must be noted that personalized treatments based on molecular medicine are usually very time-consuming and expensive, since they require a complete line of multiple diagnostic techniques in order to treat the right patient with the right drug. It is questionable, whether the health systems will accept the costs of each single molecular treatment or whether a market will be created that is big enough for current economic strategies of big pharmaceutical companies. The production of multipersonalized targeting and treatment particles could become reachable and affordable by creating a library of targeting molecules with a common linker for nanoparticle binding.

The use of SPIONs is also expected to facilitate the combination of MDT with magnetic hyperthermia, overcoming one of the biggest drawbacks of the heat treatment: The necessity of intratumoral application of the nanoparticles and an inappropriate distribution of the particles and the heat within the tumors. This approach is currently in focus of SEON in cooperation with other groups $[105,106]$.

Another anticancer therapy option that could be combined with MDT is boron neutron capture therapy. The lack of knowledge of the of boron content at a specific time point in the tumor area, which is one of the biggest obstacles for a successful application of this ther- 
apy, could be solved by the magnetic accumulation of boron-containing substances coupled to SPIONS [107].

Finally, the same strategy could further enhance the effectivity of photodynamic therapy, at the same time reducing the enduring phototoxic side-effects of this therapy.

Besides these emerging techniques MDT can be combined with all existing therapeutic strategies and applied with a therapeutic, a neoadjuvant and a palliative goal.

We are convinced that, MDT very well can be combined with a moderate standard chemotherapy, as it is expected to be well tolerated, and only a small intervention is needed for the treatment, which does not interfere with current clinical protocols.

In the neoadjuvant and palliative settings this could also be an advantage over tumor resections, especially if they have to be performed in delicate regions of the body, or would leave a big wound, which has to be closed before additive chemotherapy or radiotherapy can be administered.

In our opinion, these combinations can dramatically improve the clinical outcome of cancer therapy and could have an immense positive impact on the quality of life in cancer patients.

Apart from being a drug delivery platform, SPIONs offer the possibility of creating a powerful theranostic tool, because they can be visualized with different imaging techniques. They have been used as contrast agents for magnetic resonance imaging (MRI), giving a negative contrast in T2-sequences. This property can be utilized for a direct control of the nanoparticle distribution after MDT, showing the effectivity of the accumulation in the tumor region (see Supplementary Video). Hence, this technique could be used for either the distribution control after MDT or for an early prognosis of the therapeutic outcome, thus allowing an early decision-making for the following treatment.

Another imaging modality in particular for hospitals that have no MRI available, is ultrasound. Utilizing a kind of magnetically induced elastography, the distribution of the SPIONs could be imaged with this commonly available technique.

Finally, SPIONs are the tracers for an imaging technique, which is currently under development, namely magnetic particle imaging (MPI). Using MPI, magnetic nanoparticles can be imaged with a high temporal and spatial resolution without interference from the surrounding tissue. Additionally, the amount of nanoparticles can be simultaneously quantified. This could be of great importance for MDT, as the MRI picture could be complemented with quantitative information from MPI, which would consequently improve the prognostic value of the imaging after MDT.
Taken together, the use of magnetic particles and MDT can not only improve the effectivity of cancer treatment, but also reduce the systemic side-effects of chemotherapy. MDT can be utilized as a platform technology, as it allows combination with further treatment approaches. To perform the first successful clinical trials respective funding is necessary and we are strongly convinced that this will pave the road for an efficient emerging therapy for patients suffering from primary tumors and/or life threatening metastases. We hope that these studies will then act as a nucleus for first clinical treatment facilities and from there spreading to hospitals worldwide.

\section{Supplementary data}

To view the supplementary data that accompany this paper, please visit the journal website at: www.futuremedicine.com/ doi/full/10.2217/NNM.15.159

\section{Acknowledgements}

The authors would like to thank Dörfler, Uder, Engelhorn and Struffert (Dep. of Radiology/Neuroradiology, University Hospital Erlangen), Dörje (Pharmacy Dep., University Hospital Erlangen, Germany), Lee (Chair for Pharmaceutical Technology, Friedrich Alexander University, Erlangen, Germany), Hermann (Dep. of Internal Medicine 3, University Hospital Erlangen, Germany), Odenbach (Technical University of Dresden, Germany),Trahms (Physikalisch Technische Bundesanstalt Berlin, Germany), Vekas (Romanian Academy-Timisoara Branch, Romania), Turcu (National Institute for Research and Development of Isotopic and Molecular Technologies, Romania), Tombacz (University of Szeged, Hungary) for excellent collaboration and fruitful discussions.

\section{Financial \& competing interests disclosure}

The authors would like to thank the Else Kröner-Fresenius Stiftung (Bad Homburg v.d.H., Germany), the German Research Foundation (DFG), the German Ministry for Education and Research (BMBF), the European Union (FP7), the Bavarian State Ministry for the Environment and Consumer Protection, the Wilhelm Sander-Foundation (Munich, Germany), the Margarete Ammon-Foundation (Munich, Germany), the University Hospital Erlangen (IZKF and ELAN-Program) and the Medizinische Forschungsstiftung, (Erlangen, Germany) for funding. The authors have no other relevant affiliations or financial involvement with any organization or entity with a financial interest in or financial conflict with the subject matter or materials discussed in the manuscript apart from those disclosed.

No writing assistance was utilized in the production of this manuscript.

\section{Open access}

This work is licensed under the Attribution-NonCommercialNoDerivatives 4.0 Unported License. To view a copy of this license, visit http://creativecommons.org/licenses/by-nc-nd/4.0/ 
Executive summary

Nanomedicine offers tremendous opportunities for the development of novel therapeutic and diagnostic tools for cancer

- Effective cancer treatment remains one of the most important challenges in medicine worldwide. Due to their specific features, nanoparticles and especially superparamagnetic iron oxide nanoparticles (SPIONs) offer tremendous opportunities for new therapeutic and diagnostic tools.

Magnetic drug targeting

- Magnetic drug targeting (MDT) comprises the accumulation of drugs, which are bound to SPIONs, in a diseased body region by using a strong external magnetic field.

Requirements for clinical studies

- In Europe and USA, regulatory agencies (EMA and US FDA, respectively), have issued distinct regulatory requirements to be fulfilled if a medicinal product or drug is intended for human application. However, addressing these standard procedures may not be enough to successfully translate complex nanoparticle formulations into clinical studies.

Iron oxide nanoparticle synthesis \& coating

- The main synthesis routes for SPIONs are coprecipitation in water and thermal decomposition in boiling organic solvents. Section of experimental oncology and nanomedicine (SEON) favors coprecipitation method, as it is sufficiently reproducible and can be more easily transferred to Good manufacturing practice-conform standard procedures.

Physicochemical characterization

- Clinical translation of MDT requires a thorough physicochemical characterization of the nanoparticles with sophisticated methods. This comprises the investigation of, for example, chemical and colloidal stability in complex fluids and in the presence of a strong magnetic field, drug loading and drug release. Additionally, aging effects during different storage conditions have to be considered.

Biophysical interplay of SPIONs and the magnetic field

- To effectively accumulate SPIONs in vivo, their interplay with magnetic fields must be analyzed. Although in silico studies claim that the accumulation of SPIONs against the flow forces in the circulation is not possible, in vitro experiments demonstrated that it is indeed possible. Cross-talk between in silico and in vitro studies could further improve mathematical simulations, and offer an optimized treatment with MDT in the future.

Biological characterization \& nanotoxicology/biocompatibility

- Biological characterization and nanotoxicological studies are of crucial importance for clinical application of SPIONs. Nevertheless, many available studies are missing critical information and the standard assays are not suitable for testing SPIONs. Therefore SEON is currently establishing standard operation procedures for nanotoxicological testing that can be used for addressing SPION-related safety issues.

In vivo application

- In vivo application of MDT is crucial for demonstrating the efficacy. For a successful translation into the clinics, the right animal model must be accompanied by an appropriate application environment. SEON favores intraarterial MDT and demonstrated in 2013 in the worldwide largest preclinical animal study for MDT to date, the great potential of this emerging technique.

\section{Conclusion: the SEON-concept}

- The SEON-concept is dedicated to bringing MDT in combination with SPIONs into the clinic for a more efficient cancer treatment with significantly reduced side-effects. The central point is the combination of chemical, pharmaceutical, physical, biological, nanotoxicological and medical expertise within one group. The short communication distance and direct feedback loops between the different subunits are created in order to accelerate the translation of MDT into a clinically relevant treatment option improving the outcomes of cancer patients, as well as their quality of life.

\section{References}

1 Ferlay J, Steliarova-Foucher E, Lortet-Tieulent J et al. Cancer incidence and mortality patterns in Europe: estimates for 40 countries in 2012. Eur. J. Cancer 49(6), 1374-1403 (2013).

2 Who. Globocan 2012: Estimated Cancer Incidence, Mortality and Prevalence Worldwide in 2012. http://globocan.iarc.fr/Pages/fact_sheets_cancer.aspx

3 Langer T, Am Zehnhoff-Dinnesen A, Radtke S, Meitert J, Zolk O. Understanding platinum-induced ototoxicity. Trends Pharmacol. Sci. 34(8), 458-469 (2013).
4 Khanna R, Anger C. Patterns of patients stopping their anti-cancer drug due to its associated side effects in France, Germany, Italy, Spain and UK (EU5). Presented at: ESMO 2014. Madrid, Spain, 26-30 September 2014 (Abstract 1551P).

5 Freeman MW, Arrott A, Watson JHL. Magnetism in medicine. J. Appl. Phys. 31(5), S404-S405 (1960).

6 Gupta AK, Gupta M. Synthesis and surface engineering of iron oxide nanoparticles for biomedical applications. Biomaterials 26(18), 3995-4021 (2005). 
7 Amstad E, Textor M, Reimhult E. Stabilization and functionalization of iron oxide nanoparticles for biomedical applications. Nanoscale 3(7), 2819-2843 (2011).

8 Laurent S, Saei AA, Behzadi S, Panahifar A, Mahmoudi M. Superparamagnetic iron oxide nanoparticles for delivery of therapeutic agents: opportunities and challenges. Expert Opin. Drug Deliv. 11(9), 1449-1470 (2014).

9 Alexiou C, Jurgons R, Schmid RJ et al. Magnetic drug targeting: biodistribution of the magnetic carrier and the chemotherapeutic agent mitoxantrone after locoregional cancer treatment. J. Drug Target. 11(3), 139-149 (2003).

10 Pelgrift RY, Friedman AJ. Nanotechnology as a therapeutic tool to combat microbial resistance. Adv. Drug Deliv. Rev. 65(13-14), 1803-1815 (2013).

11 Bunjes H. Lipid nanoparticles for the delivery of poorly water-soluble drugs. J. Pharm. Pharmacol. 62(11), 1637-1645 (2010).

12 Li R, Lim S-J, Choi H-G, Lee M-K. Solid lipid nanoparticles as drug delivery system for water-insoluble drugs. J. Pharm. Invest. 40, 63-73 (2010).

13 Usha YN, Angel TT, Udupa N. Nanotechnology: perspectives on solubility/bioavailability enhancement. Pharma Rev. 8(45), 59-66 (2010).

14 Pankhurst QA, Thanh NKT, Jones SK, Dobson J. Progress in applications of magnetic nanoparticles in biomedicine. J. Phys. D Appl. Phys. 42(22), 224001 (2009).

15 Storm G, Belliot SO, Daemen T, Lasic DD. Surface modification of nanoparticles to oppose uptake by the mononuclear phagocyte system. Adv. Drug Deliv. Rev. 17(1), 31-48 (1995).

16 Brigger I, Morizet J, Laudani L et al. Negative preclinical results with stealth $((\mathrm{R}))$ nanospheres-encapsulated Doxorubicin in an orthotopic murine brain tumor model. J. Control. Release 100(1), 29-40 (2004).

17 Bilkenroth U, Taubert H, Riemann D, Rebmann U, Heynemann H, Meye A. Detection and enrichment of disseminated renal carcinoma cells from peripheral blood by immunomagnetic cell separation. Int. J. Cancer 92(4), 577-582 (2001).

18 Taupitz M, Wagner S, Hamm B, Dienemann D, Lawaczeck R, Wolf K. J. MR Lymphography using iron oxide particles. Detection of lymph node metastases in the VX2 rabbit tumour model. Acta Radiol. 34 10-15 (1993).

19 Harisinghani MG, Barentsz J, Hahn PF et al. Noninvasive detection of clinically occult lymph-node metastases in prostate cancer. N. Engl. J. Med. 348(25), 2491-2499 (2003).

20 Gaglia JL, Harisinghani M, Aganj I et al. Noninvasive mapping of pancreatic inflammation in recent-onset Type-1 diabetes patients. Proc. Natl Acad. Sci. USA 112(7), 2139-2144 (2015).

21 Alexiou C, Arnold W, Klein RJ et al. Locoregional cancer treatment with magnetic drug targeting. Cancer Res. 60(23), 6641-6648 (2000).

22 Pankhurst QA, Connolly J, Jones SK, Dobson J. Applications of magnetic nanoparticles in biomedicine. J. Phys. D Appl. Phys. 36(13), R167-R181 (2003).
23 Lyer S, Schreiber E, Tietze R et al. Nanotechnology and cancer treatment: magnetic nanoparticles for a new and innovative drug delivery system. Anticancer Res. 31(5), 1991-1992 (2011).

24 Tietze R, Lyer S, Durr S et al. Efficient drug-delivery using magnetic nanoparticles - biodistribution and therapeutic effects in tumour bearing rabbits. Nanomed. Nanotechnol. 9(7), 961-971 (2013).

25 Alexiou C. Target tumor therapy with "magnetic drug targeting": therapeutic efficacy and biokinetic study of ferrofluid bound mitoxantrone. Clin. Cancer Res. 7(11), s3704-s3705 (2001).

26 Polyak B, Friedman G. Magnetic targeting for site-specific drug delivery: applications and clinical potential. Expert Opin. Drug Deliv. 6(1), 53-70 (2009).

27 Widder KJ, Senyei AE, Ranney DF. Magnetically responsive microspheres and other carriers for the biophysical targeting of antitumor agents. Adv. Pharmacol. Chemother. 16, 213-271 (1979).

28 Widder KJ, Morris RM, Poore GA, Howard DP, Senyei AE. Selective targeting of magnetic albumin microspheres containing low-dose doxorubicin: total remission in Yoshida sarcoma-bearing rats. Eur. J. Cancer Clin. Oncol. 19(1), 135-139 (1983).

29 Lubbe AS, Bergemann C, Huhnt W et al. Preclinical experiences with magnetic drug targeting: tolerance and efficacy. Cancer Res. 56(20), 4694-4701 (1996).

30 Lubbe AS, Bergemann C, Riess $\mathrm{H}$ et al. Clinical experiences with magnetic drug targeting: a phase I study with 4 '-epidoxorubicin in 14 patients with advanced solid tumors. Cancer Res. 56(20), 4686-4693 (1996).

31 Alexiou C, Arnold W, Klein RJ et al. Locoregional cancer treatment with magnetic drug targeting. Cancer Res 60(23), 6641-6648 (2000).

32 Alexiou C, Diehl D, Henninger P et al. A high field gradient magnet for magnetic drug targeting. IEEE Trans. Appl. Supercond. 16(2), 1527-1530 (2006).

33 Agency EM. Guideline on the requirements to the chemical and pharmaceutical quality documentation concerning investigational medicinal products in clinical trials. EMEA 03/2006 (Directive CHMP/QWP/185401/2004 final). http://ec.europa.eu/health/files/eudralex

34 Agency EM. Directive EMEA/CHMP/79769/2006: Reflection paper on nanotechnology-based medicinal products for human use, (EMEA 06/2006). www.ema.europa.eu/docs/en_GB/document_library

35 Agency EM. Directive EMA/484400/2014: Mandate of the EMA Innovation Task force (ITF). (08/2014). www.ema.europa.eu/docs/en_GB/document_library

36 Laurent $\mathrm{S}$, Forge $\mathrm{D}$, Port $\mathrm{M}$ et al. Magnetic iron oxide nanoparticles: synthesis, stabilization, vectorization, physicochemical characterizations, and biological applications. Chem. Rev. 108(6), 2064-2110 (2008).

37 Yu WW, Falkner JC, Yavuz CT, Colvin VL. Synthesis of monodisperse iron oxide nanocrystals by thermal decomposition of iron carboxylate salts. Chem. Commun. (Camb.) (20), 2306-2307 (2004). 
38 Xie J, Wang J, Niu G et al. Human serum albumin coated iron oxide nanoparticles for efficient cell labeling. Chem. Commun. (Camb.) 46(3), 433-435 (2010).

39 Gonzales M, Mitsumori LM, Kushleika JV, Rosenfeld ME, Krishnan KM. Cytotoxicity of iron oxide nanoparticles made from the thermal decomposition of organometallics and aqueous phase transfer with Pluronic F127. Contrast Media Mol. Imaging 5(5), 286-293 (2010).

40 Albanese A, Tang PS, Chan WC. The effect of nanoparticle size, shape, and surface chemistry on biological systems. Annu. Rev. Biomed. Eng. 14 1-16 (2012).

41 Mahmoudi M, Saeedi-Eslami SN, Shokrgozar MA et al. Cell "vision": complementary factor of protein corona in nanotoxicology. Nanoscale 4(17), 5461-5468 (2012).

42 Gebauer JS, Malissek M, Simon S et al. Impact of the nanoparticle-protein corona on colloidal stability and protein structure. Langmuir 28 (25), 9673-9679 (2012).

43 Unterweger $\mathrm{H}$, Tietze R, Janko C et al. Development and characterization of magnetic iron oxide nanoparticles with a cisplatin-bearing polymer coating for targeted drug delivery. Int. J. Nanomedicine 9 3659-3676 (2014).

44 Kamat M, El-Boubbou K, Zhu DC et al. Hyaluronic acid immobilized magnetic nanoparticles for active targeting and imaging of macrophages. Bioconj. Chem. 21(11), 2128-2135 (2010).

45 Zaloga J, Janko C, Nowak J et al. Development of a lauric acid/ albumin hybrid iron oxide nanoparticle system with improved biocompatibility. Int. J. Nanomedicine 9, 4847-4866 (2014).

46 Bica D, Vekas L, Avdeev MV et al. Sterically stabilized water based magnetic fluids: synthesis, structure and properties. J. Magn. Magn. Mater. 311(1), 17-21 (2007).

47 Laurent S, Dutz S, Hafeli UO, Mahmoudi M. Magnetic fluid hyperthermia: focus on superparamagnetic iron oxide nanoparticles. Adv. Colloid Interface Sci. 166(1-2), 8-23 (2011).

48 Colvin VL. The potential environmental impact of engineered nanomaterials. Nat. Biotechnol. 21(10), 1166-1170 (2003).

49 Banerjee R, Katsenovich Y, Lagos L, Mciintosh M, Zhang $\mathrm{X}, \mathrm{Li}$ CZ. Nanomedicine: magnetic nanoparticles and their biomedical applications. Curr. Med. Chem. 17(27), 3120-3141 (2010).

50 Reddy LH, Arias JL, Nicolas J, Couvreur P. Magnetic nanoparticles: design and characterization, toxicity and biocompatibility, pharmaceutical and biomedical applications. Chem. Rev. 112(11), 5818-5878 (2012).

51 Tombácz E, Tóth IY, Nesztor D et al. Adsorption of organic acids on magnetite nanoparticles, $\mathrm{pH}$-dependent colloidal stability and salt tolerance. Colloids Surf. Physicochem. Eng. Aspects 435(0), 91-96 (2013).

52 Angelova A, Angelov B, Garamus VM, Couvreur P, Lesieur S. Small-angle x-ray scattering investigations of biomolecular confinement, loading, and release from liquid-crystalline nanochannel assemblies. J. Phys. Chem. Lett. 3(3), 445-457 (2012).

53 Nowak J, Wiekhorst F, Trahms L, Odenbach S. The influence of hydrodynamic diameter and core composition on the magnetoviscous effect of biocompatible ferrofluids. J. Phys. Condes. Matter 26(17), 7 (2014).

54 Eberbeck D, Lange A, Hentschel M. Identification of aggregates of magnetic nanoparticles in ferrofluids at low concentrations. J. Appl. Crystallogr. 36 1069-1074 (2003).

55 Jurgons R, Seliger C, Hilpert A, Trahms L, Odenbach S, Alexiou C. Drug loaded magnetic nanoparticles for cancer therapy. J. Phys. Condens. Matter 18(38), S2893-S2902 (2006).

56 Wiekhorst F, Seliger C, Jurgons R et al. Quantification of magnetic nanoparticles by magnetorelaxometry and comparison to histology after magnetic drug targeting. J. Nanosci. Nanotechnol. 6(9-10), 3222-3225 (2006).

57 Wiekhorst F, Liebl M, Steinhoff U, Trahms L, Lyer S, Duerr $S$, Alexiou C. Magnetorelacometry for in-vivo quantification of magnetic nanoparticle distributions after magnetic drug tarbeting in a rabbit carcinoma model. Presented at: $2 n d$ International Workshop on Magnetic Particle Imaging IWMPI 2012. Lübeck, Germany, 15-16 March 2012.

58 Richter H, Kettering M, Wiekhorst F, Steinhoff U, Hilger I, Trahms L. Magnetorelaxometry for localization and quantification of magnetic nanoparticles for thermal ablation studies. Phys. Med. Biol. 55(3), 623-633 (2010).

59 European Comission. Comission directive 2003/63/ EC of the European Pariament and of the Concil on the Community code relating to medicinal products for human use.

http://ec.europa.eu/health/documents/eudralex/vol-1

60 Zaloga J, Janko C, Agarwal R et al. Different storage conditions influence biocompatibility and physicochemical properties of iron oxide nanoparticles. Int. J. Mol. Sci. 16(5), 9368-9384 (2015).

61 Cherry EM, Maxim PG, Eaton JK. Particle size, magnetic field, and blood velocity effects on particle retention in magnetic drug targeting. Med. Phys. 37(1), 175-182 (2010).

62 Cherry EM, Eaton JK. A comprehensive model of magnetic particle motion during magnetic drug targeting. Int. J. Multiphase Flow 59, 173-185 (2014).

63 David AE, Cole AJ, Chertok B, Park YS, Yang VC. A combined theoretical and in vitro modeling approach for predicting the magnetic capture and retention of magnetic nanoparticles in vivo. J. Control. Release 152(1), 67-75 (2011).

64 Heidsieck A, Vosen S, Zimmermann K, Wenzel D, Gleich B. Analysis of Trajectories for Targeting of Magnetic Nanoparticles in Blood Vessels. Mol. Pharm. 9(7), 2029-2038 (2012).

65 Gitter K, Odenbach S. Quantitative targeting maps based on experimental investigations for a branched tube model in magnetic drug targeting. J. Magn. Magn. Mater. 323(23), 3038-3042 (2011).

66 Seliger C, Jurgons R, Wiekhorst F et al. In vitro investigation of the behaviour of magnetic particles by a circulating artery model. J. Magn. Magn. Mater. 311(1), 358-362 (2007).

67 Lyer S. TR, Jurgons R, Richter H, Wiekhorst F, Schwarz K, Trahms L, Alexiou C. Distribution of magnetic nanoparticles after magnetic drug targetin in an ex vivo bovine artery 
model. Presented at: World Congress 2009. Munich, 7-12 September 2009. Magnetorelaxometric quantification of magnetic nanoparticles in an artery model after ex vivo magnetic drug targeting. Phys. Med. Biol. 54(18), N417-N424 (2009).

69 Tietze R, Rahn H, Lyer $\mathrm{S}$ et al. Visualization of superparamagnetic nanoparticles in vascular tissue using $\mathrm{X}$ mu CT and histology. Histochem. Cell Biol. 135(2), 153-158 (2011).

70 Cicha I, Beronov K, Ramirez EL et al. Shear stress preconditioning modulates endothelial susceptibility to circulating TNF-alpha and monocytic cell recruitment in a simplified model of arterial bifurcations. Atherosclerosis 207(1), 93-102 (2009).

71 Matuszak J, Zaloga J, Friedrich RP et al. Endothelial biocompatibility and accumulation of SPION under flow conditions. J. Magn. Magn. Mater. 380, 20-26 (2015)

72 Haynes CL. The emerging field of nanotoxicology. Anal. Bioanal. Chem. 398(2), 587-588 (2010).

73 Paasonen L, Laaksonen T, Johans C, Yliperttula M, Kontturi K, Urtti A. Gold nanoparticles enable selective light-induced contents release from liposomes. J. Control. Release 122(1), 86-93 (2007).

74 Wickstrom SA, Lange A, Hess MW et al. Integrin-linked kinase controls microtubule dynamics required for plasma membrane targeting of caveolae. Dev. Cell 19(4), 574-588 (2010).

75 Andes D, Diekema DJ, Pfaller MA, Bohrmuller J, Marchillo $\mathrm{K}$, Lepak A. In vivo comparison of the pharmacodynamic targets for echinocandin drugs against Candida species. Antimicrob. Agents Chemother. 54(6), 2497-2506 (2010).

76 Hristozov DR, Gottardo S, Critto A, Marcomini A. Risk assessment of engineered nanomaterials: a review of available data and approaches from a regulatory perspective. Nanotoxicology 6, 880-898 (2012). interactions: are current in vitro tests reliable? Nanomedicine (Lond.) 6(5), 837-847 (2011)

78 Davoren M, Herzog E, Casey A et al. In vitro toxicity evaluation of single walled carbon nanotubes on human A549 lung cells. Toxicol. In Vitro 21(3), 438-448 (2007).

79 Monteiro-Riviere NA, Inman AO, Zhang LW. Limitations and relative utility of screening assays to assess engineered nanoparticle toxicity in a human cell line. Toxicol. Appl. Pharmacol. 234(2), 222-235 (2009).

80 Hoskins C, Cuschieri A, Wang L. The cytotoxicity of polycationic iron oxide nanoparticles: common endpoint assays and alternative approaches for improved understanding of cellular response mechanism. J. Nanobiotechnol. 10, 15 (2012).

81 Garcia SN, Gutierrez L, Mcnulty A. Real-time cellular analysis as a novel approach for in vitro cytotoxicity testing of medical device extracts. J. Biomed. Mater. Res. A 101(7), 2097-2106 (2013)

Durr S, Janko C, Lyer S et al. Magnetic nanoparticles for cancer therapy. Nanotechnol. Rev. 2(4), 395-409 (2013).
83 Durr S, Lyer S, Mann J et al. Real-time cell analysis of human cancer cell lines after chemotherapy with functionalized magnetic nanoparticles. Anticancer Res. 32(5), 1983-1989 (2012).

84 Janko C, Durr S, Munoz LE et al. Magnetic drug targeting reduces the chemotherapeutic burden on circulating leukocytes. Int. J. Mol. Sci. 14(4), 7341-7355 (2013).

85 Friedrich RJ C, Pöttler M, Tripal P, Zaloga J, Nowak J, Odenbach S, Liebl M, Trahms L, Stapf M, Hilger I, Lyer $S$, Alexiou C. Flow cytometry for intracellular SPION quantification: specificity and sensitivity in comparison with spectroscopic methods. Int. J. Nanomedicine 10, 4185-4201 (2015).

86 Hoehn M, Kustermann E, Blunk J et al. Monitoring of implanted stem cell migration in vivo: a highly resolved in vivo magnetic resonance imaging investigation of experimental stroke in rat. Proc. Natl Acad. Sci. USA 99(25), 16267-16272 (2002).

87 Foroutan F, Jokerst JV, Gambhir SS, Vermesh O, Kim HW, Knowles JC. Sol-gel synthesis and electrospraying of biodegradable ( $\mathrm{P} 2 \mathrm{O} 5) 55-(\mathrm{CaO}) 30-\left(\mathrm{Na}_{2} \mathrm{O}\right) 15$ glass nanospheres as a transient contrast agent for ultrasound stem cell imaging. ACS Nano 9(2), 1868-1877 (2015).

88 Morimoto Y, Okumura M, Sugibayashi K, Kato Y. Biomedical applications of magnetic fluids II. 1) preparation and magnetic guidance of magnetic albumin microsphere for site specific drug delivery in vivo. J. Pharmacobiodyn. 4(8), 624-631 (1981).

89 Osato T, Ito Y. In vitro cultivation and immunofluorescent studies of transplantable carcinomas $\mathrm{Vx} 2$ and $\mathrm{Vx} 7$. Persistence of a Shope virus-related antigenic substance in the cells of both tumors. J. Exp. Med. 126(5), 881-886 (1967)

90 Shope RE, Hurst EW. Infectious papillomatosis of rabbits: with a note on the histopathology. J. Exp. Med. 58(5), 607-624 (1933).

91 Zheng LF, Li YJ, Wang $\mathrm{H}$ et al. Combination of vascular endothelial growth factor antisense oligonucleotide therapy and radiotherapy increases the curative effects against maxillofacial VX2 tumors in rabbits. Eur. J. Radiol. 78(2), 272-276 (2011).

92 Mast TD, Barthe PG, Makin IRS et al. Treatment of rabbit liver cancer in vivo using miniaturized image-ablate ultrasound arrays. Ultrasound Med. Biol. 37(10), 1609-1621 (2011).

93 Bruners P, Braunschweig T, Hodenius $\mathrm{M}$ et al. Thermoablation of malignant kidney tumors using magnetic nanoparticles: an in vivo feasibility study in a rabbit model. Cardiovasc. Intervent. Radiol. 33(1), 127-134 (2010).

94 Lyer S, Tietze R, Jurgons R et al. Visualisation of tumour regression after local chemotherapy with magnetic nanoparticles - a pilot study. Anticancer Res. 30(5), 1553-1557 (2010).

95 Alexiou C, Arnold W, Hulin P et al. Magnetic mitoxantrone nanoparticle detection by histology, $\mathrm{x}$-ray and MRI after magnetic tumor targeting. J. Magn. Magn. Mater. 225(1-2), 187-193 (2001).

96 Alexiou C, Jurgons R, Schmid R et al. [Magnetic Drug Targeting - a new approach in locoregional tumor therapy 
with chemotherapeutic agents. Experimental animal studies]. HNO 53(7), 618-622 (2005).

97 Alexiou C, Jurgons R, Schmid R et al. In vitro and in vivo investigations of targeted chemotherapy with magnetic nanoparticles. J. Magn. Magn. Mater. 293(1), 389-393 (2005).

98 Alexiou C, Jurgons R, Seliger C, Iro H. Medical applications of magnetic nanoparticles. J. Nanosci. Nanotechnol. 6(9-10), 2762-2768 (2006).

99 Alexiou C, Jurgons R, Seliger C, Brunke O, Iro H, Odenbach S. Delivery of superparamagnetic nanoparticles for local chemotherapy after intraarterial infusion and magnetic drug targeting. Anticancer Res. 27(4A), 2019-2022 (2007).

100 Rahn H, Alexiou C, Trahms L, Odenbach S. 3-Dimensional quantitative detection of nanoparticle content in biological tissue samples after local cancer treatment. J. Magn. Magn. Mater. 360 92-97 (2014).

101 Rahn H, Gomez-Morilla I, Jurgons R, Alexiou C, Odenbach $\mathrm{S}$. Microcomputed tomography analysis of ferrofluids used for cancer treatment. J. Phys. Condens. Matter 20(20), $204152(2008)$.
102 Tietze R, Schreiber E, Lyer S, Alexiou C. Mitoxantrone loaded superparamagnetic nanoparticles for drug targeting: a versatile and sensitive method for quantification of drug enrichment in rabbit tissues using HPLC-UV. J. Biomed. Biotechnol. 2010597304 (2010).

103 Cicha I, Garlichs Christoph D, Alexiou C. Cardiovascular therapy through nanotechnology - how far are we still from bedside? Eur. J. Nanomed. 6(2), 63 (2014).

104 Durr S, Bohr C, Pottler M et al. Magnetic Tissue engineering for voice rehabilitation - first steps in a promising field. Anticancer Res. 34(11), 6806-6806 (2014).

105 Lyer S, Janko C, Tietze R et al. Hyperthermia in targeted tumour therapy using magnetic nanoparticles - first results. Anticancer Res. 34(11), 6835-6836 (2014).

106 Durr S, Schmidt W, Janko C et al. A novel magnetic field device for inducing hyperthermia using magnetic nanoparticles. Biomed. Tech (Berl.) doi:10.1515/bmt-20134129 (2013) (Epub ahead of print).

107 Unterweger $\mathrm{H}$, Tietze $\mathrm{R}$, Taccardi $\mathrm{N}$ et al. Boron containing magnetic nanoparticles for neutron capture therapy - an innovative approach for specifically targeting tumors. Anticancer Res. 34(11), 6848-6849 (2014). 